Deborah L. Johanson*, Ho Seok Ahn, Craig J. Sutherland, Bianca Brown, Bruce A. MacDonald, Jong Yoon Lim, Byeong Kyu Ahn, and Elizabeth Broadbent

\title{
Smiling and use of first-name by a healthcare receptionist robot: Effects on user perceptions, attitudes, and behaviours
}

https://doi.org/10.1515/pjbr-2020-0008

Received July 27, 2019; accepted December 20, 2019

\begin{abstract}
Robots are now starting to be developed and used as receptionists in health applications. In this regard, it is important that robots' behavioural skills are developed and researched so that people have appropriate and comfortable interactions. Smiling and use of first name are two more important social communication skills used during human interactions. While smiling and use of first name are often employed by robots in human interactions, the effect of these behaviours on perceptions of receptionist robots has not yet been experimentally investigated. This study explored the effects of robot smiling and robot use of the participant's first name on perceptions of robot friendliness, mind, and personality, as well as attitudes and smiling behaviour. Forty participants interacted with a medical receptionist robot four times, in a two by two repeated measures design. Both smiling and use of first name had significant positive effects on participants' perceptions of robot personality. Robot smiling also showed significant effects on participants' overall attitudes towards robots, ratings of robot friendliness, and perceptions of the robot's mind, and increased the frequency of participants' own smiling. There were no significant interaction effects. Robot smiling in particular can enhance user perceptions of robots and increase reciprocal smiling.
\end{abstract}

Keywords: robot, medical, receptionist, friendliness, mind perception, attitudes, personality

\footnotetext{
*Corresponding Author: Deborah L. Johanson: Department of Psychological Medicine, The University of Auckland, Auckland, New Zealand; The Centre for Automation and Robotic Engineering Science (CARES), The University of Auckland, New Zealand; E-mail: d.johanson@auckland.ac.nz

Ho Seok Ahn, Craig J. Sutherland, Bruce A. MacDonald, Jong Yoon Lim, Byeong Kyu Ahn: Department of Electrical and Computer Engineering, The University of Auckland, Auckland, New
}

\section{Introduction}

The use of robots in socially assistive roles is a growing area of research with the last two decades seeing the introduction of socially assistive robots into home and healthcare environments $[1,2]$. More recently, researchers have turned their attention to the use of social robots as receptionists within medical settings such as hospitals [3]. The application of robots as receptionists in healthcare environments requires careful consideration, given that interaction with potentially vulnerable individuals will be almost certain. In order for robots to be effective in their interactions, researchers argue that they should not only be able to perform a range of appropriate tasks [4], but that these tasks should be performed in a way that is both acceptable and comfortable [5]. To date, much of the research in healthcare robotics has focussed on developing the technical capabilities of robots.

It is fundamental to study the key social communication behaviours that a healthcare robot can display to patients and staff to establish rapport. Insight into these behaviours may be gained through the study of social communication behaviours in human social interactions, particularly interactions between medical receptionists and patients. Smiling and use of first name are two key human social behaviours that may prove important when considering the behavioural design of social robots, particularly medical receptionist robots. This paper presents research that investigates the effects of social behaviours used by a medical receptionist robot on users' perceptions and behaviour. The paper first describes background research on personality and social behaviours that humans use in

\footnotetext{
Zealand; The Centre for Automation and Robotic Engineering Science (CARES), The University of Auckland, New Zealand Bianca Brown, Elizabeth Broadbent: Department of Psychological Medicine, The University of Auckland, Auckland, New Zealand; The Centre for Automation and Robotic Engineering Science (CARES), The University of Auckland, New Zealand
} 
social settings, and then describes previous research on these social behaviours in human-robot interaction. Next, background research on medical receptionist behaviour is described, followed by research on this behaviour in robots. The aims of the study are then presented.

\section{Background}

\subsection{Personality, social behaviours, and the medical receptionist}

When considering the 'Big Five' human personality traits, the most important for positive social interactions, is the 'agreeableness' or 'friendliness' dimension [6-8]. This dimension refers to the motivation of an individual to have positive interactions with others [9]. Individuals high in the agreeableness/friendliness trait tend to be described by others as kind, caring, sympathetic, helpful, and considerate [10] while those low in this trait tend to be described as irritable, distrustful, and cold-hearted [11]. In the context of healthcare, research has found that patients and family members feel it is important that healthcare providers display friendliness traits such as kindness, care, and helpfulness [12-14].

One of the easiest and most universally understood ways in which humans demonstrate friendliness to others is through the use of smiling [15]. Smiling is one of the most studied facial expressions, with research in this area highlighting the 'power' of a smile in increasing perceptions of attractiveness, sincerity, intelligence, warmth, likability and friendliness, as well as in eliciting helping behaviour from others [15-18]. In healthcare research, smiling and friendliness have been found to positively increase patient perceptions of nurses' skills [19]. Smiling by healthcare professionals has also been associated with improvement in activities of daily living for elderly patients [20]. The $a b$ sence of smiling, along with behaviours such as looking away, was strongly associated with both short and long term decreases in physical and cognitive functioning [20]. Even in studies examining the use of smiling by a virtual human or avatar, smiling was associated with increase human smiling [21], increased human perceptions of positive affect, and increased human perceptions of the agent's social presence [22]. Smiling, therefore, represents a basic social behaviour that should be explored in terms of its usefulness in healthcare robots.

Considering how important the use of smiling is in communicating friendliness, warmth, and sincerity to others [15-18], it is perhaps surprising that little research has been done on the effect of robot smiling in human-robot interactions. Although researchers have explored how smiling affects individuals' perceptions of robot happiness [23] and overall ratings of human-robot interactions [24], no study could be found that has examined the effect of robot smiling on perceptions of robot friendliness or robot personality, and none in the area of medical receptionist robots.

When considering appropriate social behaviours for a medical receptionist robot, research on the behaviours of human medical receptionists may prove informative. A medical receptionist is the first point of contact for patients seeking medical attention, and his/her tasks range from basic administration, to management of complex patient behaviours and emotions [25-27]. Medical receptionists are expected to interact appropriately with a variety of challenging individuals, such as patients with severe mental health issues, cognitive impairments, and patients who are angry or verbally abusive [26, 27]. Human medical receptionists need to demonstrate appropriate social behaviours to build rapport with patients.

Only one study to date has specifically examined verbal communication behaviours used by medical receptionists when interacting with patients [28]. This study analysed over 283 conversations between medical receptionists and patients. It identified three distinct conversational approaches used by receptionists; a 'conversationally polite' approach, a 'task-centred' approach, or a 'rapport building' approach. The 'rapport-building' approach was the most successful. In this approach, receptionists would personalise their verbal behaviour when talking to each patient, for example, using the patient's first name. Failure of medical receptionists to include rapportbuilding aspects of communication into their dialogue often resulted in negative perceptions by patients.

As the capabilities of social robots have increased, so too has research on the application of social robots in receptionist roles $[29,30]$. Researchers have mainly explored interactions between humans and non-medical robot receptionists and investigated the robot's perceived sociability and functionality [29-31]. Variables examined have included perceived robot friendliness [29], speech recognition [31] and conversational dialogue [30].

The use of socially assistive robots in healthcare or medical environments has investigated the application of social robots as companions within elderly care facilities [1, 2] and as 'nurses' within medical environments [32]. However, research examining the application of a social robot in the role of a healthcare or medical receptionist is limited. Researchers have begun to explore how a medical robot receptionist would integrate into a medical en- 
vironment [33], and how user perceptions may affect acceptance of a robot medical receptionist [34]. However, to our knowledge research has not yet investigated how the social behaviours of a medical robot receptionists may affect user perceptions. In addition, no paper could be found specifically examining the effect of a robot, of any kind, using a person's first name on user perceptions of robot friendliness or personality. As the use of a patient's first name is an important behaviour in building rapport in interactions between human medical receptionists and patients, it represents a potentially critical social behaviour for robot medical receptionists, and another novel area of study in healthcare robotics.

Another issue to consider in studies with humanlike robots is the Uncanny Valley. Uncanny valley theory (named after the valley-like 'dip' observable in the diagram created by Mori) posits that humans will respond more positively to a robot as it becomes more human-like [5, 35]. However, when the robot reaches a point where it appears very human-like but continues to display robotic behaviours, human observers will regard the robot as eerie or repulsive. This will be explored further in the discussion when interpreting our results.

\subsection{Aim and hypotheses}

This study aimed to investigate the effects of a medical receptionist robot using smiling behaviours and a person's first name on participants' perceptions of robot friendliness. We chose use of smiling and use of first name due to their positive effects on rapport and friendliness in human social and healthcare interactions [15-20, 28]. These behaviours were also selected because they have not previously been examined in the context of a humanrobot interaction in healthcare. The research therefore makes a novel and salient contribution to the humanrobot-interaction literature.

It was hypothesised that robot smiling and use of the person's first name would result in increased ratings of robot friendliness, more positive attitudes towards the robot, better perceptions of its personality and capabilities, and more positive behaviours (participant smiling) during interactions with the robot.

\section{Method}

\subsection{Setting and participants}

This study was conducted at the University of Auckland. We recruited students using flyers placed on campuses, as well as email advertisements. Inclusion criteria included the ability to communicate fluently in English and an age of over 16 years. Ethics approval was given by The University of Auckland Human Participants Ethics Committee and written informed consent was obtained from all participants. 40 students took part in our study (35 undergraduate and 5 post-graduate students). The majority of the sample was female $(26 / 40,65 \%)$ and the mean age of participants was 24.35 years $(S D=5.49)$.

\subsection{Procedure}

The robot used for this study was the EveR-4 or 'EveR' robot. The EveR-4 robot is a female android type robot designed and created by researchers at the Korean Institute of Industrial Technology. EveR-4 is the fourth generation in the EveR series. EveR-4 is able to exhibit a wide range of facial expressions due to a pliable silicone jelly 'skin' that lies over thirty motors acting as 'facial muscles' (see Figure 1). EveR-4 has three motors that enable movement of her 'neck'. EveR-4 is able to speak and respond to statements or questions directed towards her.

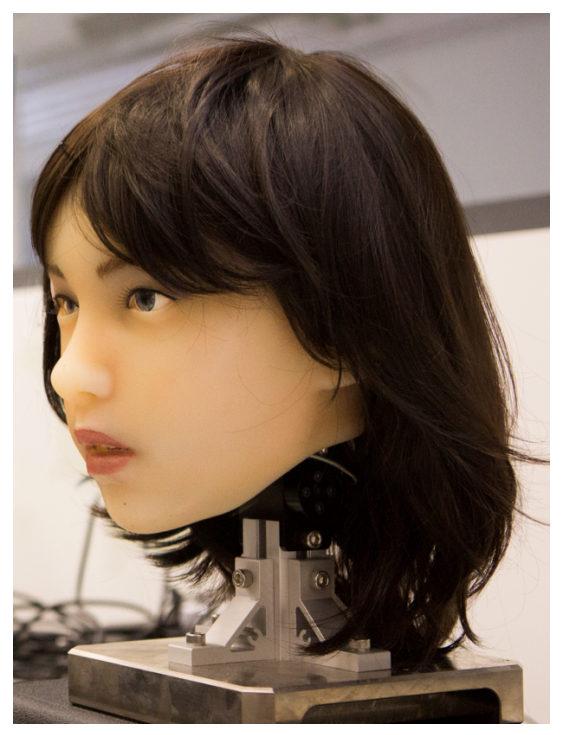

Figure 1: The Ever-4 (“EveR”) robot. 
We used a repeated measures experimental design. Each participant took part in a session lasting approximately one hour. Due to technical limitations with the robot platform, this experiment was run as a Wizard-ofOz study. Before beginning the interactions, the researcher would obtain written consent from participants and ask them to complete a baseline demographics questionnaire assessing age, gender, ethnic group, highest educational qualification, and current mood. The researcher would then provide participants with a written description of the receptionist robot (EveR) and a script to follow during the interaction. Immediately prior to the start of the interaction, the researcher would move away from the participant to sit behind a screen located behind the participant, remaining there for the duration of the interaction. A second researcher (the 'Wizard'), seated at the computer (see Figure 4) would set up the interaction from the computer and would remotely control the robot during the interaction, manually selecting the robots' responses. This second researcher did not look at the participant, nor make conversation with them for the entire duration of the experimental session. Participants interacted with EveR four times in total. For each of the four interactions, EveR was programmed with one of the following behaviours:

1. Smiling but no use of the participant's first name.

2. Use of the participant's first name but no smiling.

3. Smiling and use of participant's first name.

4. Neutral condition (i.e. no smile, no use of the participant's first name).

In interactions in which the EveR robot smiled, it smiled periodically at set times during the conversation and the rest of the time it had a neutral facial expression (see Figure 2). In non-smiling conditions, the robot kept a neutral facial expression throughout the conversation. We randomised the order of the four interactions, in other words the order in which EveR demonstrated the above four behaviours, for each participant to reduce order effects. The EveR robot demonstrated blinking and maintained eye contact with participants during all interactions.

Prior to the start of the interaction, we asked participants to imagine that they were at their local General Practitioner's (family physician) office and that EveR was the robot receptionist who was assisting them with their enquiries. Participants followed a scripted interaction, in which they asked the EveR robot to: 1) help them check in for their doctors appointment, 2) inform them regarding the wait time for their appointment, 3) help them make payment following their appointment, 4) help them book another doctors appointment, and 5) give them directions

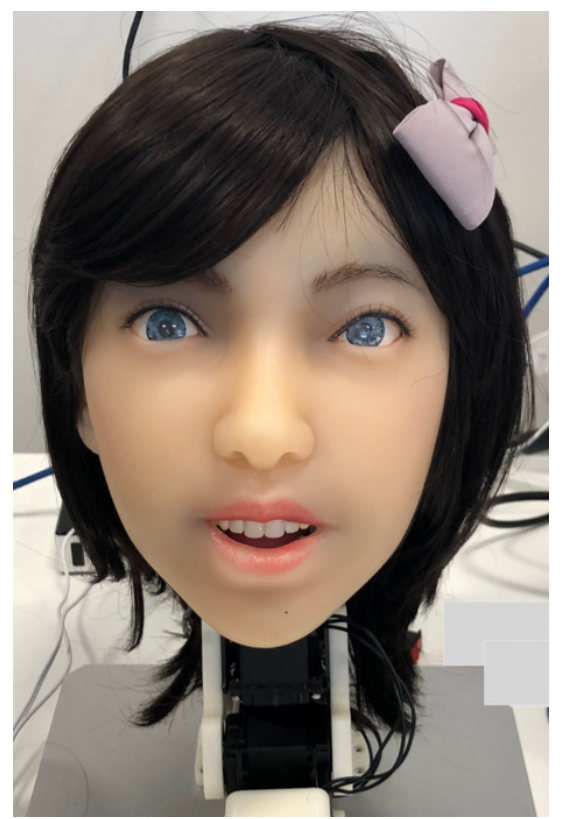

Figure 2: The EveR-4 (“EveR”) robot smiles.

to the local pharmacy. All of EveR's responses to participants were identical in each condition. We made the decision to use a scripted interaction and control EveR's responses in order to minimise any confounding variables (such as length of interaction or differences in robot and/or participant speech). During the interaction, participants sat at a table, across from the EveR robot (see Figure 3). For an image of the experimental room set-up, please see Figure 4.

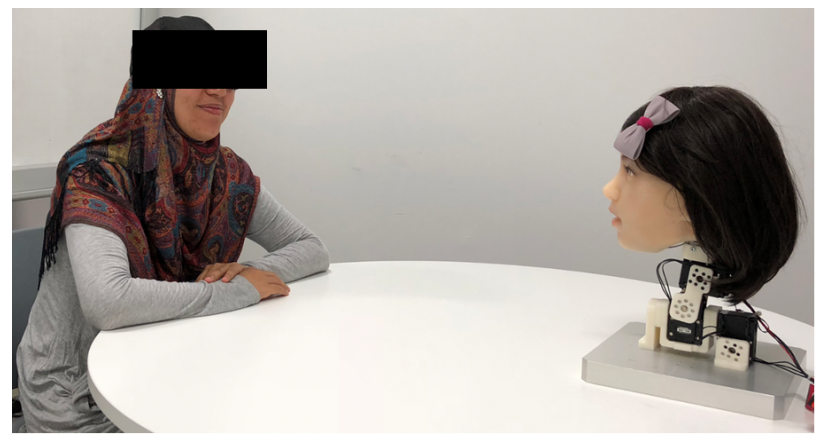

Figure 3: Participant interacts with the EveR-4 ("EveR") robot.

After each interaction with EveR, participants completed an identical questionnaire (see Section 3.3). The interactions between the participants and the EveR robot were recorded using a video camera. Participants were given a $\$ 25$ shopping voucher for their time. 


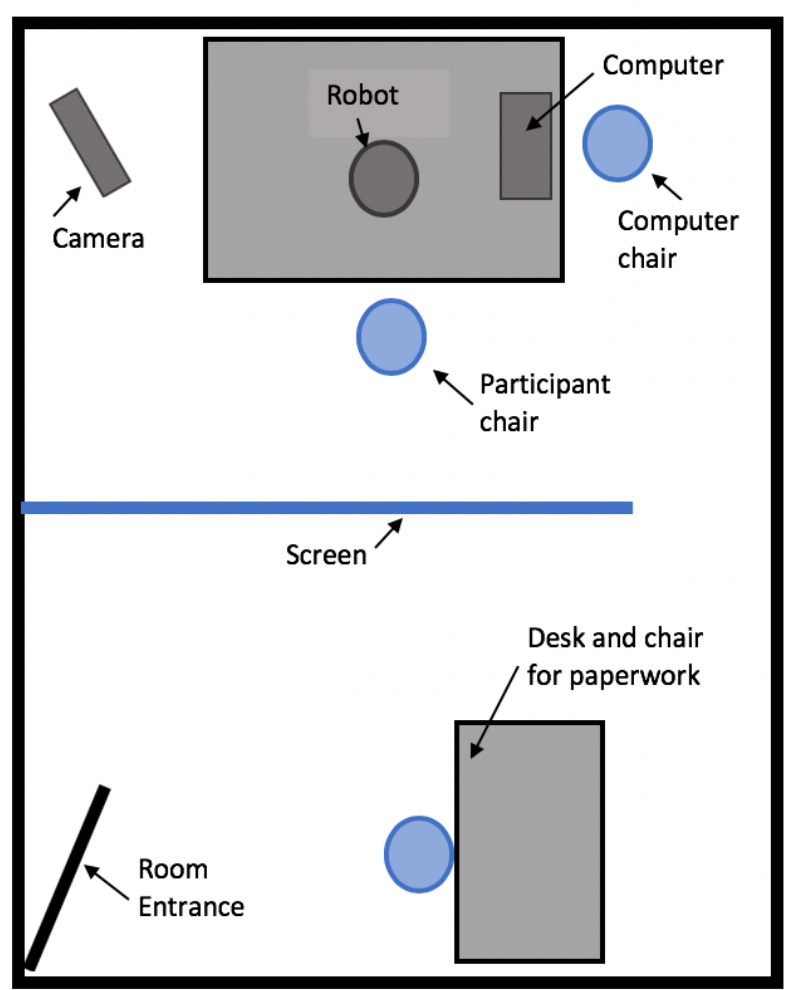

Figure 4: Experimental layout.

\subsection{Measures}

A range of measures were used to asses participants' attitudes and behaviours towards the robot as well as their perceptions of the robot's friendliness, mind, personality, and frequency of smiling, as described below.

Friendliness: The friendliness of the EveR- 4 robot was assessed using a modified 20 item version of the questionnaire used in a previous robotics study [36]; (see Appendix 1). The questionnaire contained 12 adapted items from the McGill Friendship Questionnaire (MFQ) [37], 2 adapted items from the MFQ-Friendship-Functions questionnaire [38], 3 adapted items from the Acquaintance Description Form-F2 (ADF-F2) [39], and 3 adapted items from the Interactant Satisfaction Survey (ISS) [24, 40]. For example, "I feel like Eve could be a friend of mine". Items were scored on a five-point Likert scale, with responses ranging from 'strongly agree' (5) to 'strongly disagree'(1). All of the scales included in the questionnaire have been shown to be valid and reliable measures of friendliness [36, 38-40]. A reliability analysis of the 20 item scale showed a Cronbach's $\alpha=0.94$, showing strong internal reliability. A principal component analysis revealed only one component. An inspection of the scree plot showed a clear break after the first component. There- fore, all 20 items from the Friendliness scale were summed together as a total friendliness score, where higher scores represented more friendliness.

Attitudes towards EveR-4 robot: Participant attitudes towards the EveR-4 robot were measured using the 11 item Robot Attitude Scale (RAS) [41]. The RAS asks participants to rate their agreement regarding certain attributes on an eight-point scale; where higher scores represent more negative attitudes (for example, "I think Eve was": 1 = trustworthy and $8=$ untrustworthy). This scale has been shown to have good validity in previous research [42]. Scores for the 11 items were added to create an overall attitude score. An additional question was added to this scale (human vs. robot-like) and was analysed separately.

Mind perception: The extent to which participants thought EveR could think and feel was assessed using the dimensions of Mind Perception Questionnaire [43]. For example, "How much is EveR-4 robot capable of conveying thoughts and feelings to others?" Participants are required to indicate their agreement on a seven-point scale (for example, 1 = cannot communicate and $7=$ can communicate). Six items were used. The first three items were summed together to create a sub-scale named 'agency', and the second three items were summed together to create a second sub-scale named 'experience'. This questionnaire has been shown to be a valid measure in previous research with robots [42]. Higher scores mean higher ratings of mind perception.

Personality: Asch's personality scale [44] was included in the post-interaction questionnaire, asking participants to circle one word out of 15 word pairs, that they felt most applied to the EveR4 robot (for example, 'sociable - unsociable'). Each of the two words from a single word pair was coded with either a 1 or 2 . A coding of 1 was given to the more 'positive' personality trait of the two words (e.g. sociable), while a coding of 2 was given to the more 'negative' personality trait of the two words (e.g. unsociable). Items were analysed separately. Asch's personality scale is valid and reliable and has been used as a measure of personality in published studies on psychology and social robotics [45-47].

Behaviours towards EveR-4 robot: Participants were filmed though-out their interactions with the EveR-4 robot. Natural smiling of participants was coded as a measure of reciprocated friendliness towards the robot. The coding of participant behaviour was based on the measure used in previous work [24], in which a robot monkey smiled at participants during a human-robot interaction. A natural smile was defined as a participant demonstrating a 'Duchenne' smile (when a participant raised the corners 
of their mouth and their cheeks to produce 'crow's feet' around the eyes) [48].

Frequency of smiling by the EveR-4 robot: Participant perceptions of EveR-4's smiling were measured after each interaction. Participants were asked to rate the appropriateness of the frequency of her smiling using a 3-point response scale, "What did you think of Eve's smiling": (1) too little, (2) just right, or (3) too much.

\subsection{Analysis}

Data analysis was conducted using the Statistical Package for Social Sciences (SPSS) version 22.0 using 0.05 as the critical significance level. Before conducting analyses, data was checked for outliers and errors. Participants' perceptions of EveR-4 robot's smiling frequency were analysed using a chi squared test for each of the four interactions. Personality was analysed using binomial regression in a mixed model GLMM. All other questionnaire data were analysed using linear mixed model analyses. The two independent factors for these analyses were whether or not the EveR- 4 robot smiled and whether or not the robot used the participant's first name; we also analysed the interaction between these two factors. We tested for order effects in these analyses using order as a factor. There were no significant order effects in any models.

\section{Results}

\subsection{Friendliness perceptions}

Smiling was found to have a significant positive effect on friendliness ratings $(F(1,153)=16.98, p<0.001)$. Participants rated EveR as significantly more friendly in conditions where EveR smiled (estimated $M=68.51, S E=1.84$, $95 \%$, CI [64.86, 72.15]) compared to conditions when smiling was absent (estimated $M=61.31, S E=1.87,95 \% \mathrm{CI}[57.62$, 65.00]). Use of participant first name had no significant effect on overall friendliness ratings $(F(1,153)=0.001, p$ $=0.973$ ), with no significant difference found in overall friendliness scores between conditions in which first name was used (estimated $M=64.94, S E=1.87,95 \%$ CI [61.25, 68.63]) compared to conditions in which first name use was absent (estimated $M=64.88, S E=1.85,95 \%$ CI [61.24, 68.52]). There was no significant interaction effect found between the two variables, $F(1,153)=0.22, p=0.640$ (see Figures 2 and 5).

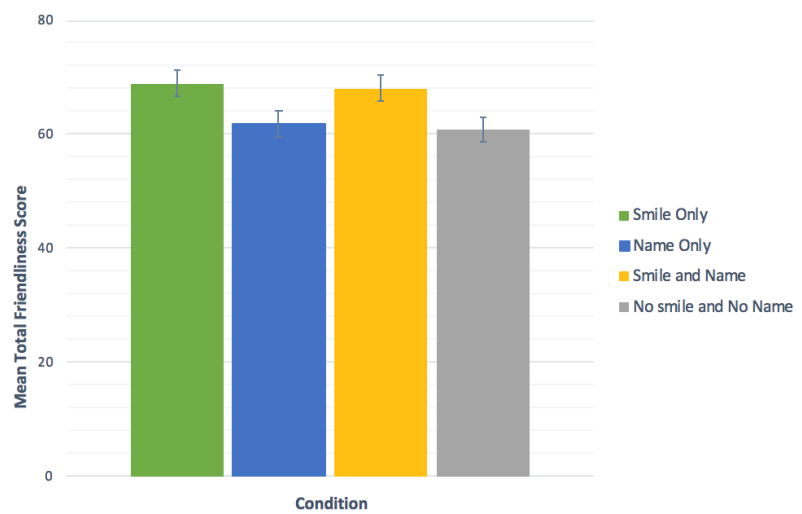

Figure 5: Effects of smiling and use of name on perceptions of robot friendliness. Note: error bars represent 1 Standard Deviation of Mean.

\subsection{Attitudes towards EveR}

The use of smiling by EveR had a significant effect on robot attitude scores $(F(1,153)=8.94, p=0.003)$, with a significant difference in scores following conditions where EveR smiled (estimated $M=35.29, S E=1.72,95 \%$ CI [31.89, 38.69]), compared to when smiling was absent (estimated $M=39.94, S E=1.74,95 \%$ CI $[36.49,43.38])$.

Use of participants' first name by EveR had no significant effect on attitude scores $(F(1,153)=0.07, p=0.798)$ with no difference found in attitude scores following conditions in which first name was used by EveR (estimated $M$ $=37.81, S E=1.74,95 \% \mathrm{CI}[34.37,41.26])$ compared to when first name was not used (estimated $M=37.42, S E=1.72,95 \%$ CI $[34.02,40.81])$. There was no interaction effect found between smiling and use of first name $F(1,153)=0.33, p=$ 0.568 .

Participants were also significantly more likely to rate EveR as more 'human-like' rather than 'machine-like' ( $F(1$, $155)=12.15, p=0.001)$ following conditions in which EveR smiled (estimated $M=4.41, S E=0.20,95 \%$ CI $[4.02,4.80]$ ) compared to conditions in which smiling was absent (estimated $M=5.39, S E=0.20,95 \%$ CI $[5.00,5.78])$.

There was no significant difference found in participant ratings of the item 'machine-like vs human-like' ( $F(1$, $155)=0.00, p=0.991$ ) following conditions where EveR used a participants' first name (estimated $M=4.90, S E=$ $0.20,95 \% \mathrm{CI}[4.51,5.30])$ compared to when first name was not used (estimated $M=4.90, S E=0.20,95 \%$ CI $[4.51,5.29]$ ). There was no significant interaction found between smiling and use of first name, $F(1,155)=0.94, p=0.334$. 


\subsection{Mind perception}

Participants perceived EveR as having significantly more agency $(F(1,155)=12.02, p=0.001)$ when she smiled (estimated $M=11.44, S E=0.40,95 \%$ CI $[10.64,12.23])$ compared to when she did not smile (estimated $M=9.45, S E=$ $0.41,95 \%$ CI $[8.65,10.25])$. EveR's use of the participant's first name had no significant effect on the robot's overall agency score $(F(1,155)=0.17, p=0.683)$ with no significant difference found between conditions in which EveR used a participant's first name (estimated $M=10.33, S E=0.41$, $95 \%$ CI $[9.53,11.13])$ compared to when first name use was absent (estimated $M=10.56, S E=0.40,95 \%$ CI [9.77, 11.36]). No significant interaction was found between smiling and use of first name $F(1,155)=0.04, p=0.849$ (see Figure 6).

Participants perceived EveR as having significantly more experience $(F(1,155)=6.97, p=0.009)$ when she smiled (estimated $M=8.35, S E=0.39,95 \%$ CI [7.57, 9.13]), compared to when she did not smile (estimated $M=6.87$, $S E=0.40,95 \% \mathrm{CI}[6.09,7.66])$. There was no significant difference found between conditions where EveR used a participants first name (estimated $M=7.62, S E=0.40,95 \% \mathrm{CI}$ $[6.84,8.41]$ ), and when she did not (estimated $M=7.60, S E$ $=0.39,95 \% \mathrm{CI}[6.82,8.38])$ in regards to overall experience score $(F(1,155)=0.002, p=0.965$. There was no significant interaction found between smiling and use of first name, $F(1,155)=0.05, p=0.824$ (see Figure 6).

\subsection{Personality}

The use of smiling by EveR had a significant effect on participants' ratings of the robot's personality. More participants rated EveR positively on six personality items (generous, happy, good-natured, sociable, humane, and warm) when she smiled compared to when she did not smile (see Table 1).

Participants rated EveR as significantly more humane when she used their first name compared to when she did not (Coefficient $=-1.38, S E=0.594, p=0.022)$. No other personality items were significantly affected by use of first name. There was no significant interaction effect found between name and smiling variables for any personality item.

\subsection{Effects of smiling on perceptions of robot behaviours}

Participant perceptions of smile frequency were significantly different following conditions where EveR smiled,

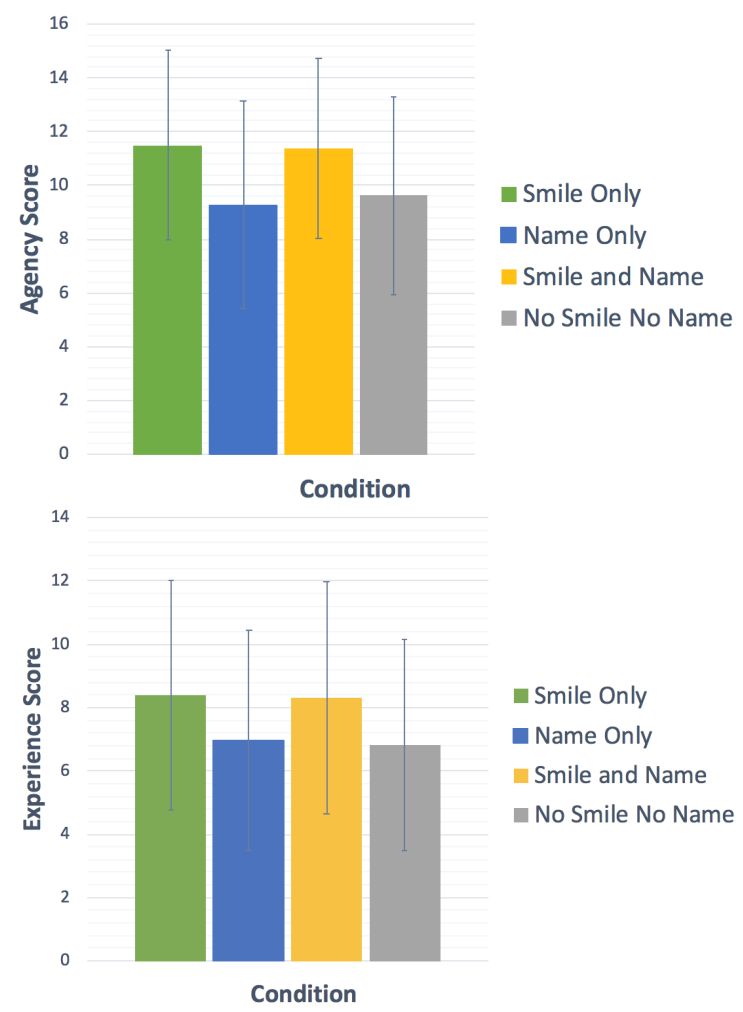

Figure 6: Effects of smiling and use of name on perceptions of robot agency and experience. Note: error bars represent 1 Standard Deviation of Mean.

compared to conditions in which smiling was not present $(F(1,154)=15.26, p<0.001)$. In trials where smiling was used, EveR was rated as smiling just the right amount of time in $60 \%$ of trials, not enough $34 \%$ of the time, and too much $6 \%$ of the time. In trials where smiling was absent, EveR was rated as smiling not enough $67 \%$ of the time, smiling just the right amount of time by $30 \%$, and too much by $3 \%$.

Perceptions of EveR's smiling were found to be unaffected by EveR's use of a participant's first name, $F(1,154)$ $=0.05, p=0.816$ ), with no significant difference in participant ratings of EveR's smile frequency between conditions in which EveR used a participant's first name and when first name was not used. There was no significant interaction effect found between the two variables, $F(1,154)=$ $0.03, p=0.861$.

\subsection{Participant's own smiling}

Participants were found to perform a significantly greater number of natural smiles $(F(1,82)=5.10, p=0.027)$ in conditions where EveR smiled (estimated $M=2.24, S E=0.39$, $95 \%$ CI $[1.47,3.02])$, as opposed to when she did not smile 
Table 1: Effect of robot smiling on participant perceptions of robot personality traits.

\begin{tabular}{|c|c|c|c|c|}
\hline \multirow[b]{2}{*}{ Personality Item } & $\begin{array}{c}\text { Model } \\
\text { (Smiling, Name, \& }\end{array}$ & \multirow[b]{2}{*}{ Coefficient } & nile & \multirow[b]{2}{*}{$p$} \\
\hline & Akaike & & $S E$ & \\
\hline Generous (vs. Ungenerous) & 752.732 & 1.91 & 0.696 & 0.007 \\
\hline Happy (vs. Unhappy) & 737.933 & 1.25 & 0.594 & 0.037 \\
\hline Good-natured (vs. Irritable) & 314.538 & 1.61 & 0.341 & $<0.001$ \\
\hline Humorous (vs. Humourless) & 799.798 & -0.03 & 0.686 & 0.966 \\
\hline Sociable (vs. Unsociable) & 727.325 & 1.97 & 0.514 & $<0.001$ \\
\hline Important (vs. Insignificant) & 782.610 & 0.97 & 0.741 & 0.195 \\
\hline Humane (vs. Ruthless) & 782.610 & 2.00 & 0.342 & $<0.001$ \\
\hline Talkative (vs. Restrained) & 712.825 & 0.93 & 0.572 & 0.106 \\
\hline Warm (vs. Cold) & 665.924 & 1.22 & 0.492 & 0.015 \\
\hline Wise (vs. Shrewd) & 732.606 & 0.03 & 0.637 & 0.962 \\
\hline Popular (vs. Unpopular) & 669.268 & 0.83 & 0.469 & 0.078 \\
\hline Good Looking (vs Unattractive) & 673.486 & 0.54 & 0.474 & 0.257 \\
\hline Persistent (vs. Unstable) & 775.140 & 0.82 & 0.669 & 0.225 \\
\hline Frivolous (vs. Serious) & 859.582 & -0.78 & 0.757 & 0.304 \\
\hline Altruistic (vs. Self-centred) & 733.562 & 0.852 & 0.672 & 0.206 \\
\hline Imaginative(vs. Hard-headed) & 711.907 & 0.55 & 0.536 & 0.308 \\
\hline Strong (vs. Weak) & 684.498 & 0.40 & 0.524 & 0.450 \\
\hline Honest (vs. Dishonest) & 870.551 & 0.43 & 0.956 & 0.651 \\
\hline
\end{tabular}

Note: Bolded $p$ value indicated a significance of $p<0.05 . M=$ mean. $S E=$ standard error.

(estimated $M=1.35, S E=0.39,95 \%$ CI $[0.57,2.13])$. Use of name had no significant effect on smiling $(F(1,82)=0.060$, $p=0.805)$, with no significant difference in smile frequency found between conditions where EveR used a participant's name (estimated $M=1.75, S E=0.40,95 \%$ CI [0.95, $2,54]$ ), and when she did not use a name (estimated $M=$ $1.85, S E=0.38,95 \%$ CI $[1.08,2.71])$. There was no significant interaction effect found between the two variables, $(F(1,82)=0.24, p=0.626)$.

\section{Discussion}

In this study we examined the effect of a robot medical receptionist's social communication behaviours, specifically that of smiling and use of first name, on human perceptions, attitudes, and behaviours. As hypothesised, smiling by the EveR-4 robot resulted in significantly higher ratings of robot friendliness, more positive attitudes towards EveR, better perceptions of the robot's personality, and greater perceptions of the robot's agency and experience. In addition, we found that participants smiled significantly more often in conditions where EveR smiled. We further found that participants rated the frequency of the robot's smiling as more appropriate in the smiling conditions.

Contrary to hypotheses, use of the participant's first name had no effect on perceptions of friendliness or attitudes towards the robot. Use of first name also had no effect on participant perceptions of the robot's agency or experience. Use of name did, however, result in participants being significantly more likely to rate the robot as humane (compared to ruthless).

\subsection{Comparisons with prior work}

As discussed, prior research in human behaviour has found smiling to be associated with increased positive perceptions, such as sincerity, intelligence, warmth, likability and friendliness [15-18]. Our results reflect these findings, with participants rating the robot as significantly more friendly, happy, good natured, experienced, and warm when it smiled. EveR was also rated as being significantly higher in both agency and experience, in conditions in which she smiled as opposed to those conditions in which she did not. Appropriate use of smiling by healthcare professionals has been found to be associated with higher patient perceptions of skill level and friendliness [16, 19]. It 
may be that the use of smiling by the EveR robot, caused participants to perceive EveR as more skilled and friendly, resulting in more positive ratings across a number of variables.

Similar to our findings, previous research has shown that smiling by a virtual agent increased human smiling [21]. Smiling by an avatar was also associated with increased human perceptions of the avatar's social presence [22]. These findings are also supported by our research, as participants rated the EveR robot higher in both experience and agency, in conditions where the robot smiled as opposed to conditions where she did not. In addition, the EveR robot was rated as significantly more sociable (versus unsociable) following conditions in which it smiled.

In regards to robotics research in particular, this is the first study to show that robot smiling can significantly increase ratings of robot friendliness, personality, agency, and experience. This is also the first study to show that robot smiling can significantly increase human smiling. Although prior robotics research did find that robot smiling increased participant smiling, these findings were not statistically significant [24].

Use of first name by the robot resulted in significantly more participants rating the robot as humane. In research by Hewitt et al. [29] involving human medical receptionists, a personalised approach, such as using a patient's first name, resulted in increased rapport between the medical receptionist and patient. It might be that the robot's use of a participant's first name increased rapport, resulting in higher perceptions of humanity.

\subsection{Contribution to existing literature}

This research highlights the importance of smiling as a basic social behaviour that should be considered in the design of social robots. Although many engineers already include robot smiling as a default social behaviour, this is the first study to produce evidence relating to the significant effect robot smiling has on user perceptions of robot friendliness, personality, attributes, and capabilities. Although the robot's use of a participant's first name did not influence many of the outcomes measured, it did positively increase participant perceptions of the robot being humane. This is another valuable and novel finding in regards to robot social communication behaviours and an important social skill for use by receptionist robots.

\section{Limitations}

Limitations of this study include the repeated measures design, which can cause practice or fatigue effects. To reduce the likelihood of order effects we randomised the order of the conditions. When analysing results, we found that order effects were not significant in any models. Future research could replicate this study using a between groups design in order to corroborate findings. Additional limitations include the use of a student sample and experimental setting. Due to these limitations the results of this study may not generalise to a patient population in a real clinic. Finally, as the EveR robot is a 'head and neck' robot without a body, it is important to recognise that different results may have been found had a 'full-bodied' robot been used. While it is not unusual for researchers to use 'head and neck' robots in studies examining human-robot interactions $[24,49,50]$, funding restraints dictated the decision to use a 'head and neck' robot in this study.

It is important for future research to test the effects found in this study with patient populations in healthcare environments. In addition, future work could investigate whether the use of smiling and first name by a healthcare robot in situations other than those considered here, such as blood pressure checks or medication reminders, have an effect on study outcomes.

Uncanny valley theory posits that human responses to a robot will shift from positive to revulsion or 'eeriness' as the robot approaches (but fails to completely mimic) a human appearance [5, 35]. Indeed, some participants in this experiment mentioned finding the robot 'scary' or 'creepy'. If participants found the robot uncanny, this might have resulted in lower ratings and occluded some of the experimental effects. Future research therefore could replicate the study with a less human-like robot.

\section{Conclusion}

In conclusion, this study shows that both smiling and the use of the participant's first name have positive effects on perceptions of a medical receptionist robot. The effects for smiling were the strongest and affected perceptions of mind, personality, friendliness, as well as overall attitudes and smiling behaviour, whereas the use of the name only affected perceptions of robot personality. These results have implications for choice of social behaviours for robots in healthcare environments. 
Acknowledgement: This work was supported by the Technology Innovation Program (10077553, Development of Social Robot Intelligence for Social Human-Robot Interaction of Service Robots) funded by the Ministry of Trade, industry and Energy (MI, Korea).

We would also like to acknowledge the University of Auckland's Centre for Automation and Robotic Engineering Science (CARES) team for their ongoing support during this study.

\section{References}

[1] R. Bemelmans, G. J. Gelderblom, P. Jonker, and L. de Witte, "Effectiveness of robot paro in intramural psychogeriatric care: $\mathrm{A}$ multicenter quasi-experimental study," J. Am. Med. Dir. Assoc., vol. 16, no. 11, pp. 946-950, November 2015.

[2] H. Robinson, B. Macdonald, N. Kerse, and E. Broadbent, "The psychosocial effects of a companion robot: A randomized controlled trial," J. Am. Med. Dir. Assoc., vol. 14, no. 9, pp. 661-667, September 2013.

[3] "The hospitals of the future: Robo-receptionists, Al doctors and a 'Fitbit for brains'," (2017, January), Wired. Available: https://www.wired.co.uk/article/hp-re-inventors-healthhospitals-of-the-future

[4] E. Broadbent, R, Tamagawa, A. Patience, B. Knock, N. Kerse, K. Day, and B. A. Macdonald, "Attitudes towards health-care robots in a retirement village," Australas. J. Ageing, vol. 31, no. 2, pp. 115-120, June 2012.

[5] M. L. Walters, D. S. Syrdal, K. Dautenhahn, R. Te Boekhorst, and K. L. Koay, "Avoiding the uncanny valley: Robot appearance, personality and consistency of behaviour in an attentionseeking home scenario for a robot companion," Auton. Robots, vol. 24, no. 2, pp. 159-178, 2008.

[6] D. C. Dryer, "Getting personal with computers: How to design personalities for Agents," Appl. Artif. Intell., vol. 13, no. 3, pp. 273-295, 1999.

[7] D. S. Moskowitz, "Unfolding interpersonal behavior," J. Pers., vol. 73, no. 6, pp. 1607-1632, December 2005.

[8] J. S. Wiggins, "A psychological taxonomy of trait-descriptive terms: The interpersonal domain,”J. Pers. Soc. Psychol., vol. 37, no. 3, pp. 395-412, 1975.

[9] W. Tov, Z. L. Nai, and H. W. Lee, "Extraversion and agreeableness: Divergent routes to daily satisfaction with social relationships," J. Pers., vol. 84, no. 1, pp. 121-134, February 2016.

[10] W. G. Graziano and N. Eisenberg, "Agreeableness: A dimension of Personality," in Handbook of personality psychology, R. Hogan, J. A. Johnson, and S. R. Briggs (Eds.), Academic Press, 1997, pp. 795-824. Cambridge: MA. DOI 10.1016/B978012134645-4/50031-7.

[11] S. Roccas, L. Sagiv, S. H. Schwartz, and A. Knafo, "The big five personality factors and personal values," Pers. Soc. Psychol. Bull., vol. 28, no. 6, pp. 789-801, 2002.

[12] M. Clowers, "Young women describe the ideal physician," Adolescence, vol. 37, no. 148, pp. 695-704, Winter 2002. Available: https://www.questia.com/library/journal/1G197723207/young-women-describe-the-ideal-physician
[13] E. C. Meyer, M. D. Ritholz, J. P. Burns, and R. D. Truog, "Improving the quality of end-of-life care in the pediatric intensive care unit: Parents' priorities and recommendations," Pediatrics, vol. 117, no. 3, pp. 649-657, March 2006.

[14] R. P. Strauss, M. C. Sharp, C. Lorch, and B. Kachalia, "Physicians and the communications of 'bad news': parents experiences of being informed of their child's cleft lip and/or palate," Pediatrics, vol. 96, no. 1 Pt 1, pp. 82-89, 1995. Available: https://www.ncbi.nlm.nih.gov/pubmed/7596729

[15] S. de Lemus, R. Spears, and M. Moya, "The power of a smile to move you: Complementary submissiveness in women's posture as a function of gender salience and facial expression," Pers. Soc. Psychol. Bull., vol. 38, no. 11, pp. 1480-1494, November 2012.

[16] N. Gueguen and M. De Gail, "The effect of smiling on helping behaviour: Smiling and good Samaritan behaviour," Commun. Rep., vol. 16, no. 2, pp. 133-140, 2003.

[17] S. Lau, "The effect of smiling on person perception," J. Soc. Psychol., vol. 117, no. 1, pp. 63-67, 1982.

[18] H. T. Reis, I. M. Wilson, C. Monestere, S. Bernstein, K. Clark, E. Seidl, et al., "What is smiling is beautiful and good," Eur. J. Soc. Psychol., vol. 20, no. 3, pp. 259-267, 1990.

[19] P. R. Wysong and E. Driver, "Patients' perceptions of nurses' skill," Crit. Care Nurse, vol. 29, no. 4, pp. 24-37, quiz 37, August 2009. Available: https://www.ncbi.nlm.nih.gov/pubmed/ 19648596

[20] N. Ambady, J. Koo, R. Rosenthal, and C. H. Winograd, "Physical therapists' nonverbal communication predicts geriatric patients' health outcomes," Psychol. Aging, vol. 17, no. 3, pp. 443-452, September 2002.

[21] N. Kramer, S. Kopp, C. Becker-Asano, and N. Sommer, "Smile and the world will smile with you: The effects of a virtual agents smile on user evaluation and behaviour," Int. J. Hum. Comput. Stud., vol. 71, no. 3, pp. 335-349, 2013.

[22] S. Y. Oh, J. Bailenson, N. Krämer, and B. Li, "Let the avatar brighten your smile: Effects of enhancing facial expressions in virtual environments," PLoS One, vol. 11, no. 9, p. e0161794, September 2016.

[23] M. Blow, K. Dautenhahn, A. Appleby, C. L. Nehaniv, and D. Lee, "Perceptions of robot smiles and dimensions for human-robot interaction design," in ROMAN 2006 - The 15th IEEE International Symposium on Robot and Human Interactive Communication, Hatfield, UK, September, 2006, pp. 469-474.

[24] L. D. Riek, P. C. Paul, and P. Robinson, "When my robot smiles at me: Enabling human robot rapport via real time head gesture mimicry," J. Multimodal User Interfaces, vol. 3, no. 3, pp. 99-108, 2009.

[25] M. Gallagher, P. Pearson, C. Drinkwater, and J. Guy, "Managing patient demand: A qualitative study of appointment making in general practice," Br. J. Gen. Pract., vol. 51, no. 465, pp. 280-285, April 2001.

[26] P. M. Neuwelt, R. A. Kearns, and A. J. Browne, "The place of receptionists in access to primary care: Challenges in the space between community and consultation," Soc. Sci. Med., vol. 133, no. 1, pp. 287-295, May 2015.

[27] J. Ward and R. McMurray, "The unspoken work of general practitioner receptionists: A re-examination of emotion management in primary care," Soc. Sci. Med., vol. 72, no. 10, pp. 1583-1587, May 2011. 
[28] H. Hewitt, L. McCloughan, and B. McKinstry, "Front desk talk: Discourse analysis of receptionist-patient interaction," Br. J. Gen. Pract., vol. 59, no. 565, pp. e260-e266, August 2009.

[29] A. Niculescu, B. van Dijk, A. Nijholt, D. K. Limbu, S. L. See, and A. H. Y. Wong, "Socializing with Olivia, the youngest robot receptionist outside the lab," in: S. S. Ge, H. Li, J. J. Cabibihan, and Y. K. Tan (Eds.), Social Robotics, ICSR 2010, Lecture Notes in Computer Science, vol. 6414, Springer, Berlin, Heidelberg, pp. 50-62.

[30] M. K. Lee, S. Kiesler, and J. Forlizzi, "Receptionist or information kiosk: How do people talk with a robot?," in CSCW '10: Proceedings of the 2010 ACM Conference on Computer Supported Cooperative Work, Savannah, GA, February, 2010, pp. 31-40.

[31] R. Nisimura, T. Uchida, A. Lee, H. Saruwatari, K. Shikano, and Y. Matsumoto, „ASKA: receptionist robot with speech dialogue system," in Proceedings of the 2002 IEEE/RSJ International Conference on Intelligent Robots and Systems, Lausanne, Switzerland, October, 2002, vol. 2, pp. 1314-1319.

[32] M. G. Jacob, Y. Li, and J. P. Wachs, "Gestonurse: a multimodal robotic scrub nurse," in Proceedings of the 7th ACM/IEEE International Conference on Human-Robot Interaction (HRI), Boston, MA, USA, March, 2012, pp. 153-154.

[33] H.S. Ahn, M. H. Lee, and B. A. MacDonald, "Healthcare robot systems for a hospital environment: CareBot and ReceptionBot," in Proceedings of the $24^{\text {th }}$ IEEE International Symposium on Robot and Human Interactive Communication (RO-MAN), Kobe, Japan, August, 2015, pp. 571-576.

[34] A. Gambino, J. Kim, and S. S. Sundar, "Digital doctors and robot receptionists: user attitudes that predict acceptance of automation in healthcare facilities," in CHI EA '19: Extended Abstracts of the 2019 CHI Conference on Human Factors in Computing Systems, Glasgow, Scotland, May, 2019, Paper No.: LBW0287.

[35] M. Mori, K. F. MacDorman, and N. Kageki, "The uncanny valley: From the field,” IEEE Robot. Autom. Mag., vol. 19, no. 2, pp. 98-100, 2012.

[36] A. Pereira, I. Leite, S. Mascarenhas, C. Martinho, and A. Paiva, "Using empathy to improve human-robot relationships," in Human-Robot Personal Relationships, M. H. Lamers and F. J. Verbeek (Eds.), HRPR 2010, Lecture Notes of the Institute for Computer Sciences, Social Informatics and Telecommunications Engineering, vol. 59, Springer, Berlin, Heidelberg, pp. 130-138.

[37] M. J. Mendelson and F. E. Aboud, "Measuring friendship quality in late adolescence and young adults: McGill friendship questionnaires," Can. J. Behav. Sci., vol. 31, no. 2, pp. 130-132, 1999.

[38] M. J. Mendelson and F. E. Aboud, "McGill Friendship Questionnaire - Respondent's affection (MFQ-RA)," Measurement Instrument Database for the Social Sciences, 1999. Available: http://www.midss.org/content/mcgill-friendshipquestionnaire--respondents-affection-mfq-ra

[39] P. H. Wright, "A bare-bones guide to the Acquaintance Description Form-F2," from PaulWright.com, 1997. Available: http://paulhwright.com/paulhwright/docs/ADF-F2.doc

[40] S. H. Kang, J. H. Watt, and S. K. Ala, “Communicators' perceptions of social presence as a function of avatar realism in small display mobile communication devices," in Proceedings of the 41st Annual Hawaii International Conference on System Sciences (HICSS 2008), Waikolao, HI, USA, January, 2008.

[41] E. Broadbent, R. Tamagawa, N. Kerse, B. Knock, A. Patience, and B. MacDonald, "Retirement home staff and residents' pref- erences for healthcare robots," RO-MAN 2009 - The 18th IEEE International Symposium on Robot and Human Interactive Communication, Toyoma, Japan, September, 2009.

[42] R. Q. Stafford, B. A. MacDonald, C. Jayawardena, D. M. Wegner, and E. Broadbent, "Does the robot have a mind? Mind perception and attitudes towards robots predict use of an eldercare robot," Int. J. Soc. Robot., vol. 6, no. 1, pp. 17-32, 2014.

[43] H. M. Gray, K. Gray, and D. M. Wegner, "Dimensions of mind perception," Science, vol. 315, no. 5812, pp. 619-624, February 2007.

[44] S. E. Asch, "Forming impressions of personality," J. Abnorm. Psychol., vol. 41, no. 3, pp. 258-290, July 1946.

[45] J. A. Mann, B. A. MacDonald, I. H. Kuo, X. Li, and E. Broadbent, "People respond better to robot than computer tablets delivering healthcare instructions," Comput. Human Behav., vol. 43, no. 1, pp. 112-117, 2015.

[46] L. E. Williams and J. A. Bargh, "Experiencing physical warmth promotes interpersonal warmth," Science, vol. 322, no. 5901, pp. 606-607, October 2008.

[47] E. Broadbent, V. Kumar, X. Li, J. Sollers, III, R. Q. Stafford, B. A. MacDonald, et al., "Robots with display screens: $A$ robot with a more humanlike face display is perceived to have more mind and a better personality," PLoS One, vol. 8, no. 8, p. e72589, August 2013.

[48] S. D. Gunnery and M. A. Ruben, "Perceptions of Duchenne and non-Duchenne smiles: A meta-analysis," Cogn. Emotion, vol. 30, no. 3, pp. 501-515, 2016.

[49] C. Breazeal, "Emotion and social humanoid robots," Int. J. Hum. Comput. Stud., vol. 59, no. 1-2, pp. 119-155, 2003.

[50] C. Breazeal, "Towards sociable robots," Robotics and Autonomous Systems, vol. 42, no. 3-4, pp. 167-175, 2003.

\section{Appendix 1}

\section{Friendliness Measure}

You have just completed your interaction with Eve. Based on this interaction, please indicate your agreement with the following statements.

\section{Strongly agree}

\section{Agree}

\section{Neutral}

\section{Disagree}

\section{Strongly disagree}

1. I enjoyed talking with Eve.

2. Eve helped me with this appointment.

3. Eve shared her emotions with me.

4. I would trust Eve if coming to the doctor again.

5. Eve was supportive of my needs. 
6. I felt comfortable with Eve.

7. I felt I could tell private and sensitive things to Eve.

8. Eve is genuinely likeable.

9. I felt Eve expressed feelings and emotions that were appropriate for the situation.

10. I feel like Eve could be a friend of mine.

11. I would be happy to see Eve as a receptionist again.

12. Eve's answers were helpful to me.

13. Eve showed sensitivity towards my emotions.

14. I think Eve is loyal.

15. I felt confident interacting with Eve.

16. Eve's support was useful to me.

17. I think Eve would make me feel more calm if I was nervous.

18. Eve seemed to enjoy helping me.

19. I felt like Eve was being true and sincere.

20. I would like to have a friendly chat with Eve. 